\title{
0716. Does intravenous iron induce oxydative stress in critically ill patients? A comparison with healthy volunteers
}

\author{
S Lasocki ${ }^{*}$, P Piednoir ${ }^{2}$, C Couffignal ${ }^{3}$, E Rineau ${ }^{1}$, C Schilte $^{4}$, G Dufour $^{2}$, X Duval ${ }^{4}$, F Driss $^{5}$ \\ From ESICM LIVES 2014 \\ Barcelona, Spain. 27 September - 1 October 2014
}

\begin{abstract}
Introduction
Anaemia is frequent in critically ill patients. Iron deficiency, secondary to blood losses or prior to admission, is in part responsible for this anaemia. Iron may thus be proposed to critically ill patients $(\mathrm{CI})$. However, iron may promote oxidative stress, which is potentially deleterious. In a mouse model, we previously demonstrated that iron induces less oxidative stress in inflamed mice than in control ones [1], but no data are available in the CI.
\end{abstract}

\section{Objective}

To compare the oxydative stress induced by an intravenous infusion of iron in $\mathrm{CI}$ and healthy volunteers $(\mathrm{V})$.

\section{Methods}

Adult critically ill patients (from 2 ICUs) and healthy Volunteers were included after informed consent. Blood samples were drawn before (T0) and 2, 6 and 24 hours (T2, T6, T24) after an intravenous infusion of $100 \mathrm{mg}$ of iron sucrose (Venofer@) over 60 minutes. Markers of lipid oxidation $-8 \alpha$-Isoprostanes (8ISO)-, protein oxidation -Advanced Oxydized Protein Product (AOPP)- as well as glutathion reduced/oxidized (GSH/GSSG), Nontransferrin bound iron (NTBI) have been measured at these time points. Variations of area under the curves from $\mathrm{T} 0$ to $\mathrm{T} 6\left(\triangle \mathrm{AUC}_{0-6}\right)$ have been compared using a Wilcoxon test. Data are expressed as $\mathrm{n}(\%)$, mean $\pm \mathrm{SD}$ or median[min-max].

\section{Results}

38 CI have been studied (25(66\%) males, aged 67.9[1985 ]yrs, 38(100\%) ventilated, SAPSII 48.5[21-80], Hb 8.4

\footnotetext{
${ }^{1} \mathrm{CHU}$ de Angers, Département d'Anesthésie-Réanimation, Angers, France
} Full list of author information is available at the end of the article
[6.6-11.8] g/dl) and $39 \mathrm{~V}(18(46 \%)$ males, aged 42.1[2178] yrs, Hb 13.9[11.9-17.2] g/dl). Iron treatment indications for CI were (many causes possible): 18(45\%) elevated soluble transferrin receptor (sTfR), 14(35\%) ferritin $<100 \mu \mathrm{g} / \mathrm{l}+\mathrm{TSat}<20 \%, 12(30 \%)$ blood loss $>$ one blood mass, $9(22 \%)$ elevated sTfR/log Ferritin ratio. At $\mathrm{T}_{0}$, [8ISO] was higher in CI than in $\mathrm{V}$ 8.48[3.1-63.4] vs. 4.51[2.05-13.33] pmol/l), but the $\Delta \mathrm{AUC}_{0-6 \mathrm{~h}}(8 \mathrm{ISO})$ was not different $(\mathrm{p}=0,38)$. Only the $\Delta \mathrm{AUC}_{0-6 \mathrm{~h}}(\mathrm{GSH})$ was lower in $\mathrm{V}(\mathrm{p}=0,009)$, arguing for a more important decrease in anti-oxidant defences. The table summarized all the results. Eight CI had a second set of dosages (after the $4^{\text {th }}$ iron infusion) showing no difference in any markers compared to the first set of dosages.

\section{Discussion}

We haven't seen any increase in lipid (8ISO) or protein (AOPP) oxidation in CI compared to V. On the contrary, $\mathrm{V}$ had a greater decrease in anti-oxidant (ie GSH),

Table 1 Oxidative stress markers dosages.

\begin{tabular}{|c|c|c|c|}
\hline & Critically ill $(n=38)$ & Voluntaries $(n=39)$ & $P$ \\
\hline$\triangle \mathrm{AUCO}-\mathrm{H6}$ (8ISO) & $0.06[-86.51-29.45]$ & $2.01[-16.60-17.95]$ & 0.38 \\
\hline CO AOPP $(\mu \mathrm{g} / \mathrm{l})$ & $36.57[17.46-98.90]$ & 19.07 [10.49-34.92] & \\
\hline $\begin{array}{l}\triangle A \cup C 0-H 6 \\
\text { (AOPP) }\end{array}$ & $14.8[-172.3-110.1]$ & $12.4[-92.7-63.7]$ & 0.69 \\
\hline $\mathrm{CO}$ GSH $(\mu \mathrm{g} / \mathrm{l})$ & $3.97[1.49-7.31]$ & $1.22[0.66-2.12]$ & \\
\hline$\triangle \mathrm{AUCO}-\mathrm{H} 6(\mathrm{GSH})$ & $\begin{array}{l}-8.34[-615.4- \\
329.36]\end{array}$ & $-142.4[-1640.1-989.1]$ & 0.009 \\
\hline$\overline{C O}$ GSSG $(\mu \mathrm{g} / \mathrm{l})$ & $94.90[31.80-349.00]$ & $414.2[266.3-845.5]$ & \\
\hline$\triangle \mathrm{AAUCO-H6}$ (GSSG) & $1.44[-163.17-77.26]$ & $\begin{array}{l}-11.79[-152.85 \\
-116.36]\end{array}$ & 0.43 \\
\hline CO NTBI $(\mu \mathrm{g} / \mathrm{L})$ & $0.23[-1.44-0.75]$ & $.22[-1.35-0.89]$ & \\
\hline$\overline{\triangle A U C O-H 6}(\mathrm{NTBI})$ & $0.74[-3.75-9.53]$ & $0.33\left[\begin{array}{ll}-4.53 & -9.77]\end{array}\right.$ & 0.35 \\
\hline
\end{tabular}


suggesting higher oxidative stress. Iron in the CI doesn't induce more oxidative stress than in $\mathrm{V}$, but other studies are needed to confirm the absence of clinical toxicity.

\section{Authors' details}

${ }^{1} \mathrm{CHU}$ de Angers, Département d'Anesthésie-Réanimation, Angers, France. ${ }^{2} \mathrm{CHU}$ Bichat-Claude Bernard-Paris Diderot, Departement d'Anesthésie Réanimation, Paris, France. ${ }^{3} \mathrm{CHU}$ Bichat-Claude Bernard-Paris Diderot, Biostatistiques, Paris, France. ${ }^{4} \mathrm{CHU}$ Bichat-Claude Bernard-Paris Diderot, CIC, Paris, France. ${ }^{5}$ INSERM U 1149, Unniversité Paris Nord Val de Seine, Paris, France.

Published: 26 September 2014

\section{Reference}

1. Heming CCM Crit Care Med 2012, 40(7):2141-8.

doi:10.1186/2197-425X-2-S1-P47

Cite this article as: Lasocki et al:: 0716. Does intravenous iron induce oxydative stress in critically ill patients? A comparison with healthy volunteers. Intensive Care Medicine Experimental 2014 2(Suppl 1):P47.

\section{Submit your manuscript to a SpringerOpen ${ }^{\odot}$ journal and benefit from:}

- Convenient online submission

- Rigorous peer review

- Immediate publication on acceptance

- Open access: articles freely available online

- High visibility within the field

- Retaining the copyright to your article 